Jerzy Bralczyk

\title{
ETHIK DER SPRACHE ${ }^{1}$
}

Die Medien, auch wenn sie die gesellschaftlichen Folgen verbaler Gewalt kritisieren, so exponieren sie zugleich die ausdrucksstärksten Aussagen, wodurch sie diese automatisch propagieren.

Unseren öffentlichen, insbesondere politischen Diskurs versuchen meistens zwei Metaphern zu beschreiben: Krieg und Theater. Eine Anknüpfung an nicht nur kommunikative Vergehen: Aggression und Lüge. In öffentlichen Äußerungen beobachtet man den fehlenden Willen zur Verständigung sowie mangelnde Sorgfalt im Umgang mit der Wahrheit - eine Prämisse, um die öffentliche Rede als unethisch, unredlich und politischen Interessen untergeordnet zu qualifizieren.

Die Sprache verschärft sich insbesondere dann, wenn „die politische Szene“ zur „Kampfarena“ von zwei - unterschiedlich definierten, jedoch im allgemeinen eindeutig wahrgenommenen - Lagern wird. Im Fall eines heftigen politischen Konflikts fallen dabei von beiden Seiten dieselben Bezichtigungen kommunikativer Übeltaten. Die am politischen Diskurs Beteiligten konzentrieren ihre wesentlichen Vorwürfe gegenüber den politischen Gegnern oft um deren Äußerungen herum. Eine Vielzahl verbaler Angriffe wird durch das Aufdecken der Unehrlichkeit und Aggressivität der anderen gerechtfertigt. Einen beachtlichen Teil des kommunikativen Raums scheinen aggressive und lügnerische Aussagen über vermeintliche aggressive und lügnerische Aussagen der Gegenseite einzunehmen. Dabei werden vereinfachende, sloganartige, das Verhalten des Gegners etikettierende Formeln als in jedem Falle bewiesen und einsatzbereit verwendet. Den einen kann man den Einsatz von Hassrede vorwerfen, den anderen die Industrie der Verachtung.

${ }^{1}$ Erstveröffentlichung in: Academia 1(45) 2016: 57. Aus dem Polnischen übersetzt von Jacek Makowski. Sprachliche Beratung und Korrektur von Heinrich Hofmann-Psuty. 
Der Einsatz bestimmter Wörter durch die eine Seite begrenzt deren Einsatz durch die andere Seite. So steht es beispielsweise um den Begriff der Wahrheit. Verwendet wird dieses Wort meist in dem Kontext des Kampfes, bei der Formulierung von Vorwürfen gegenüber dem Gegner (oder in dem Fall gar des Feindes), was zu mentalen Shortcuts wie die Smolensk-Wahrheit (symmetrisch etwa gegenüber der Smolensk-Lüge) veranlasst. Und die Wahrheit, die uns doch frei machen soll, muss oft schmerzhaft sein. Dabei kann unser Streben nach der Wahrheit sogar die aggressivsten Haltungen und Maßnahmen rechtfertigen. Ein derartiger Missbrauch eines der wichtigsten Werte ist ein Akt, der die ethischen Regeln der Kommunikation besonders verletzt.

Eine andere gefährliche Erscheinung ist der Einsatz von Begriffen und Wörtern, deren emotionale Konnotation gegenüber der eigentlichen denotativen Bedeutung prädominant ist und diese in den Schatten stellt - wodurch die Ausdrücke auf willkürliche Adressaten in beliebigem Kontext bezogen werden können. Ein Wort wie Kommunist hat für viele seinen klaren Bedeutungsumfang verloren. Nur einige wenige könnten heutzutage das Wort in der Bedeutung eines, auch fanatischen, Anhängers der Idee sozialer Gleichheit und Gerechtigkeit mit der allgemein gültigen Beschimpfung verbinden, doch der Ausruf Kommunisten und Diebe gilt als fast selbstverständliche Zusammenstellung und wird auch als solche Verwendet.

Das normenbildende Potenzial der öffentlichen Sprache, auch im Sinne eines Kampfschauplatzes, kann der sprachlichen Kommunikation auch im Allgemeinen schaden. Es rechtfertigt Aggression und weckt Unglauben an die Nützlichkeit der Sprache als Mittel zu guter Verständigung und zur Entschärfung von Konflikten. Rechtliche Absicherungen zur Identifizierung und Bestrafung von Sprachmissbrauch sind notwendig und nicht selten wirksam, mindestens genauso wichtig erscheint jedoch immer öfter die öffentliche Missbilligung von Verstößen gegen die Ethik von sprachlichen Handlungen. Obwohl der Missbilligung derartiger Missstände keine besondere Wirkung zugeschrieben werden kann und sich darin sogar eine gewisse Naivität entdecken lässt, würde deren Fehlen doch eigenartig und sozial schädigend wirken. Umso mehr, als Aggressivität der Sprache nicht ohne Grund von medialen Anbietern als besonders attraktiv angesehen wird. Die Medien sind nicht nur Teil der öffentlichen Auseinandersetzung, sie kreieren diese geradezu. Auch wenn sie die gesellschaftlichen Folgen verbaler Gewalt kritisieren, so exponieren sie zugleich die ausdrucksstärksten Aussagen, wodurch sie diese automatisch propagieren. 
Akzeptanz findet angesichts dieser Tatsache der Einsatz sprachmanipulativer Eingriffe, darunter nicht nur rhetorischer, sondern auch eristischer Tricks. Wir erklären uns damit einverstanden, dass unsere Konsumentscheidungen von gewieften Copywritern beeinflusst werden und uns durch den Mund des Politikers Persuasionsspezialisten, Pressesprecher oder Spindoktoren ansprechen - wobei wir es grundsätzlich vorziehen würden, von denjenigen überzeugt zu werden, die keine Experten im Überzeugen sind.

Die Identifizierung beinahe offensichtlicher und vorausgesetzter Manipulation in öffentlichem Sprachgebrauch, die auch nicht frei von unethischen Elementen ist, kann dazu führen, dass die Empfänger sogar hinter anscheinend redlichen Aussagen hinterlistige, in diesem Sinne auch aggressive und lügnerische Eingriffe vermuten. Eine Aufforderung zur Versöhnung kann so als ein perfider Akt verstanden werden, wodurch der Sender als derjenige dargestellt wird, welcher in Wirklichkeit seinen Gegner hinterhältig schwächen will.

Wer weiß? Als richtiger Ausweg aus dieser Situation könnte sich unter Umständen das Identifizieren und Propagieren von positiven Erscheinungen erweisen. Miteingerechnet wären auch solchen Aussagen, bei denen der gute Wille sowie das Streben nach Versöhnung selbst dann vorausgesetzt werden können, wenn der Verdacht legitim ist, das diese nicht vorhanden sind. 